\title{
La méthode Bilan travail pour l'approche du fonctionnement des élevages extensifs uruguayens ${ }^{*}$
}

\author{
Francisco Dieguez ${ }^{1}$ \\ Hermes Morales ${ }^{1}$ \\ Sylvie Cournut ${ }^{2}$ \\ ${ }^{1}$ Instituto Plan Agropecuario \\ Brd Artigas 3802 \\ Montevideo \\ Uruguay \\ $<$ fdieguez@planagropecuario.org.uy> \\ <fmorales@planagropecuario.org.uy> \\ ${ }^{2}$ VetAgro Sup Clermont \\ Unité mixte de recherche (UMR) Métafort \\ BP 35 \\ 63370 Lempdes \\ France \\ <s.cournut@vetagro-sup.fr>
}

\begin{abstract}
Résumé
L'élevage traditionnel extensif uruguayen doit faire face à l'accroissement des surfaces en cultures et en forêts, aux épisodes récents de sécheresse et aux bouleversements des marchés internationaux. Les institutions liées à l'élevage comme l'Instituto Plan Agropecuario (IPA), conscientes de la complexité de cette situation, cherchent à adapter leur appui technique en intégrant une composante travail dans l'approche des exploitations. Cette étude rend compte de l'expérimentation, sur huit exploitations suivies par l'IPA, de la méthode "bilan-travail" (BT) et en donne les principaux enseignements. Les trois modalités d'organisation du travail identifiées (autonomie, partage et supervision) mettent en jeu l'investissement de l'éleveur dans la réalisation des travaux et la gestion de la main-d'œuvre salariée. Si des liens entre dimensions des exploitations, système de production et modalités d'organisation du travail sont apparus, ils n'expliquent pas tout. D'autres éléments comme la volonté de travailler avec sa femme, transmettre son expérience à ses enfants, se réserver du temps pour d'autres activités, améliorer la productivité de son exploitation, doivent également être pris en compte. L'application de la méthode BT s'est avérée pertinente pour approcher le fonctionnement des exploitations et en comprendre les déterminants principaux. Donnant un nouvel éclairage des élevages, elle offre des pistes intéressantes pour l'adaptation du conseil technique.
\end{abstract}

Mots clés : élevage extensif ; organisation du travail ; Uruguay ; viande bovine.

Thèmes : économie et développement rural ; productions animales.

\section{Abstract \\ The work assessment method for a functional approach to extensive Uruguayan beef farms}

Uruguay's traditional extensive ranching must face the increase of crops and forest surface, the recent episodes of drought and the disorders on international markets. The institutions linked to agricultural systems, like the Instituto Plan Agropecuario (IPA), are conscious of the complexity of the situation and try to adapt technical advice integrating the work dimension into the farm-system approach. This study summarizes and presents the main conclusions of the Work Assessment method application on eight farms accompanied by the IPA. Three work organisational choices are distinguished (autonomy, share and supervision) considering the farmer's fieldwork participation and the wage-earning worker's administration. The results may be partially explained by some aspect such as farm size, the productive system and/or work organization choices, but other factors such as the desire to do the work in couples, to transmit work experience to children and the enhancement of the system's productivity may be also considered. The Work Assessment method is revealed to be significant for a functional approach to farm-systems and helps in understanding the main determinants of its way of functioning. This method is a new type of system approach and gives some clues for an adaptation of technical advice.

Key words: beef; extensive husbandry; Uruguay; work organization.

Subjects: animal productions; economy and rural development.

\footnotetext{
*Pour citer cet article: Dieguez F, Morales H, Cournut S. La méthode Bilan travail pour I'approche du fonctionnement des élevages extensifs uruguayens. Cah Agric $2010 ; 19$ : 316-22. DOI : 10.1684/agr.2010.0419.
} 
E n Uruguay, l'élevage des herbivores occupe historiquement une place importante non seulement dans l'économie mais aussi dans l'identité culturelle du pays. Quatre-vingt-neuf pour cent de la superficie totale du pays sont consacrés à la production de viande destinée pour l'essentiel à l'exportation (Estadísticas Agropecuarias [DIEA], 2000). Fondé quasi exclusivement sur les prairies naturelles avec un chargement animal de $0,7 \mathrm{UGB}^{1} / \mathrm{ha}$ en moyenne, l'élevage extensif uruguayen est menacé par l'accroissement des surfaces en cultures et en forêts (eucalyptus) ainsi que par les récents épisodes de sécheresse. Le prix des terres a fortement augmenté sous l'influence de nouveaux acteurs comme les fonds de pension. Qui plus est, l'essor d'entreprises agricoles capables de travailler de grandes superficies et le développement d'entreprises forestières modifient le marché de l'emploi dans les zones rurales déjà très peu peuplées (Arbeletche et Carballo, 2008).

Dans ce contexte, s'intéresser au devenir de l'élevage extensif demande de prendre en compte, dans le cadre de l'appui technique aux éleveurs, la gestion de la main-d'œuvre, la pluriactivité des ménages (20\% des élevages sont gérés par des exploitants ayant une autre activité [Riella et Mascheroni, 2008]) et la volonté de maitrise par les éleveurs de leur travail (nature du travail, qualité de vie mais aussi productivité du travail). Pour analyser le fonctionnement des exploitations d'élevage du point de vue de l'organisation du travail, l'Instituto Plan Agropecuario (IPA) a utilisé la méthode Bilan travail (BT) (Dedieu et al., 2006). Cette méthode permet de quantifier, à l'échelle d'une campagne agricole (en Uruguay, elle va du $1^{\text {er }}$ juillet au 30 juin), le travail lié à la conduite des troupeaux et des surfaces selon les catégories de main-d'œuvre et d'évaluer la marge de manœuvre en temps des exploitants pour réaliser d'autres activités, dans l'exploitation ou en dehors, et disposer de temps libre. Cette étude a permis de tester la méthode BT sur huit exploitations et rend compte des principaux enseignements tirés.

\footnotetext{
${ }^{1}$ UGB : unité gros bétail. Une unité gros bétail en Uruguay correspond à une vache non gestante et non lactante de $380 \mathrm{~kg}$ du poids vif.
}

\section{Méthode d'étude}

Les huit cas d'étude ont été choisis pour couvrir au mieux la diversité observée dans les exploitations suivies par l'IPA, qui sont des exploitations relevant du système productif extensif traditionnel, de petite ou moyenne taille. Ils sont distribués dans les différentes régions d'élevage du pays (figure 1).

Les données recueillies lors des enquêtes BT ont été traitées pour chacune des exploitations et les résultats restitués individuellement auprès de chaque éleveur. Nous avons ensuite réalisé une " analyse de groupe " (Cournut et al., 2009) afin de décrire la diversité de l'organisation du travail et d'identifier les déterminants les plus importants de celle-ci. Compte tenu des spécificités de l'élevage uruguayen, nous avons été amenés à modifier les principes d'application de cette méthode BT. En effet, l'importance, dans ce pays, de la pluriactivité et du recours au salariat, nous a conduit à définir la cellule de base comme l'ensemble des travailleurs permanents organisant le travail sur l'exploitation, qu'ils soient pluriactifs ou peu investis dans la réalisation du travail agricole, du moment qu'ils gèrent régulièrement leur exploitation en supervisant le travail de leurs salariés (les salariés, même permanents, ne font pas partie de la cellule de base). Dans la même logique, nous avons étendu la prise en compte des tâches d'astreinte au travail de supervision réalisé par l'exploitant quotidiennement ou plusieurs fois par semaine.

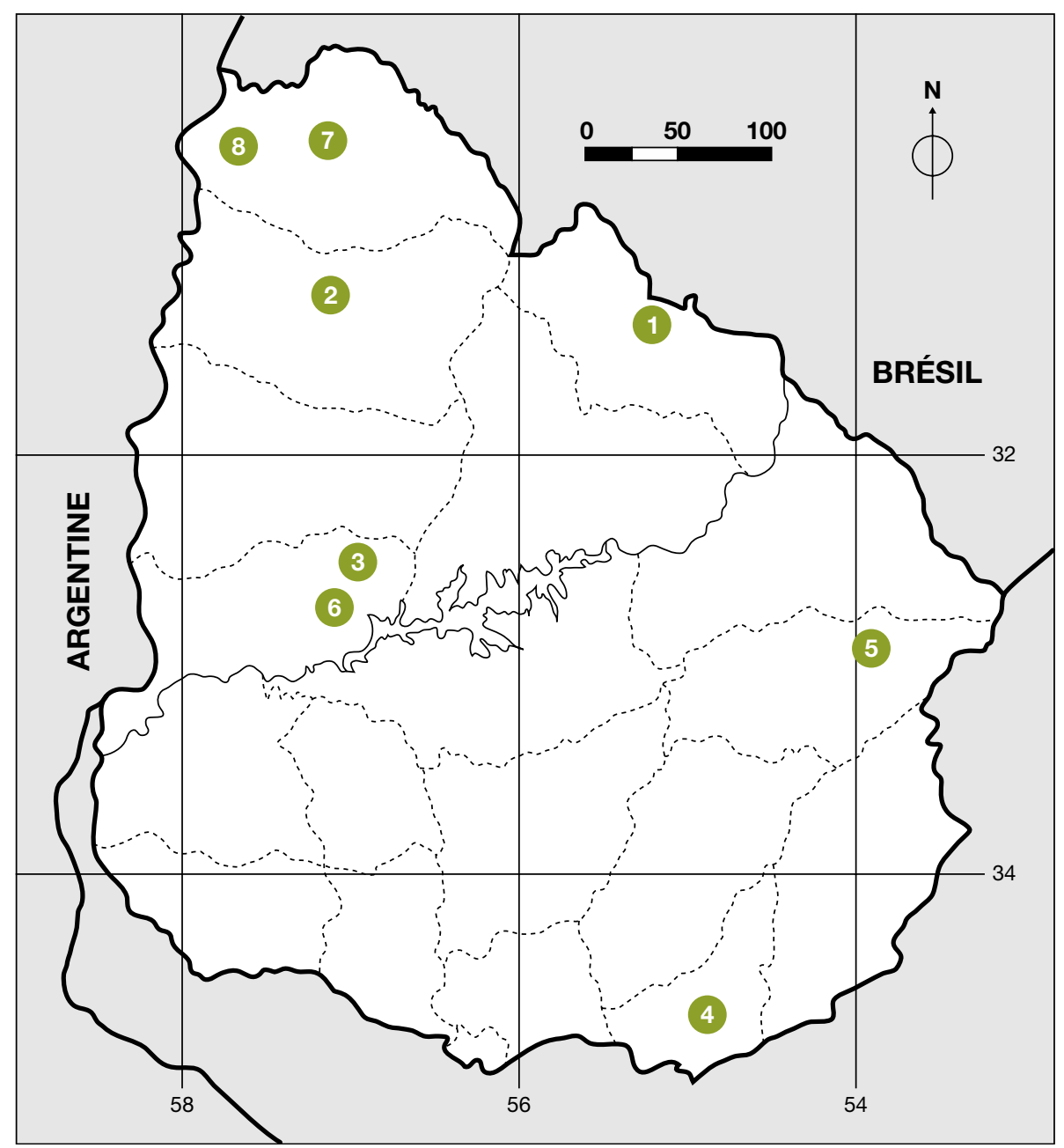

Figure 1. Répartition géographique des huit exploitations enquêtées.

Figure 1. Geographical distribution of the eight farms studied. 


\section{Résultats}

\section{Systèmes étudiés}

Comme on l'observe classiquement en Uruguay, les systèmes d'élevage sont mixtes : ils comprennent des bovins pour la viande et des ovins pour la laine et la viande, les animaux pâturant souvent ensemble (tableau 1). (Estadísticas Agropecuarias (DIEA), 2000). Sept élevages sont uniquement naisseurs pour les bovins et cinq sont naisseursengraisseurs ("ciclo completo") pour les ovins. Dans notre échantillon, seule l'exploitation E5 a une activité de finition des mâles à l'herbe, en donnant du foin pendant l'hiver, mais en Uruguay ce type de système d'alimentation ne concerne qu'un tiers des exploitations.

La ressource fourragère est surtout constituée de prairies naturelles, les prairies cultivées ne représentant que 5 à $20 \%$ de la surface. Pour profiter au mieux de la pousse de l'herbe, les vêlages sont souvent concentrés sur la fin de l'hiver austral, début du printemps. La castration et le marquage des animaux se font au moment du sevrage des veaux qui a lieu généralement vers l'âge de 6 mois. Les agnelages, concentrés aussi au printemps, sont souvent suivis de la tonte. Le principal investissement est la construction de clôtures de 7 à 8 fils non barbelés et très tendus. L'installation la plus répandue est la manga, couloir de contention avec parc d'attente utilisé pour vacciner, marquer, peser, regrouper les animaux. Seules deux exploitations (E3 et E6) ont investi dans du matériel agricole - tracteurs de haute puissance, semeuse pour le semis de prairies cultivées et faucheuse rotative pour la récolte du foin sur les prairies naturelles, par exemple - pour réaliser les travaux sur les surfaces.

\section{Main-d'œuvre}

Dans six cas sur huit, l'exploitation est gérée par le chef de famille, l'intervention de la femme étant souvent limitée au travail administratif, activité non prise en compte dans le BT (tableau 2). Dans ce cas, la cellule de base comprend une seule personne, le chef de famille, même si celui-ci exerce une autre activité (autre métier en ville pour E4 ou engagement politique important pour E6). Pour les deux autres exploitations, la cellule de base est composée de deux personnes : le couple (E2) ou le père et le fils (E7). La famille habite sur l'exploitation, sauf dans un des cas de double activité (E4) ou dans le cas de la grande exploitation (E3).

L'aide bénévole des fils des éleveurs travaillant hors exploitation est ponctuelle pour le travail de saison (traitements sanitaires du bétail, marquage, castration, sevrage) (E2, E5). L'entraide est présente

\section{Tableau 1. Description structurelle et fonctionnelle des élevages.}

Table 1. Structural and functional description of farms.

\begin{tabular}{lccrrrrrr}
\hline Exploitation & E1 & E2 & E3 & E4 & E5 & E6 & E7 & E8 \\
\hline Syst. production bovins & Naiss. & Naiss. & Naiss. & Naiss. & CC & Naiss. & Naiss. & Naiss. \\
Syst. production ovins & CC & CC & CC & néant & CC & Naiss. & CC & Naiss. \\
Surface (hectares) & 285 & 300 & 1861 & 154 & 604 & 760 & 514 & 753 \\
Surface prairies semées (\%) & 11 & 17 & 20 & 15 & 5 & 16 & 9 & 16 \\
Mères bovines & 130 & 110 & 509 & 82 & 238 & 118 & 146 & 149 \\
Mères ovines & 105 & 220 & 1595 & - & 316 & 499 & 895 & 955 \\
UGB totales & 265 & 324 & 1415 & 113 & 520 & 382 & 433 & 477 \\
Chargement (UGB totales/ha) & 0,93 & 1,08 & 0,76 & 0,73 & 0,86 & 0,50 & 0,84 & 0,63 \\
\hline
\end{tabular}

Naiss. : naisseur uniquement ; CC : cycle complet ; UGB : unité gros bétail.

\section{Tableau 2. Caractérisation du collectif de travail.}

Table 2. Work group characterization.

\begin{tabular}{|c|c|c|c|c|c|c|c|c|}
\hline Exploitation & E1 & E2 & E3 & E4 & E5 & E6 & E7 & E8 \\
\hline Nombre pCB & 1 & 2 & 1 & 1 & 1 & 1 & 2 & 1 \\
\hline Caractéristiques de la CB & $\begin{array}{l}\text { CF } \\
\text { DA }\end{array}$ & Couple & CF & $\begin{array}{l}\text { CF } \\
\text { DA }\end{array}$ & CF & CF & Père et fils & $\mathrm{CF}$ \\
\hline Âge pCB & 70 & 48 et 47 & 55 & 43 & 58 & 57 & 64 et 34 & 65 \\
\hline Nombre de salariés permanents & 1 & & 3 & 1 & 2 & 2 & 1 & 2 \\
\hline Bénévoles & & $x$ & & & $x$ & & & $x$ \\
\hline Entraide & & $x$ & & $x$ & & & $x$ & \\
\hline Salariés occasionnels & & & $x$ & $x$ & $x$ & $x$ & $x$ & $x$ \\
\hline Entreprise pour le diagnostic gestation & & $x$ & $x$ & & & & & $x$ \\
\hline Entreprise pour le semis des prairies & $x$ & $x$ & & $x$ & $x$ & $x$ & & \\
\hline Entreprise pour la tonte & $x$ & $x$ & $x$ & & $x$ & $x$ & $x$ & $x$ \\
\hline
\end{tabular}

pCB : personne de la cellule de base ; CF : chef de famille ; DA : double actif. 
dans trois exploitations pour le même type de tâches (E2, E4, E7). Dans tous les cas, la tonte est faite par une entreprise. D'autres entreprises sont engagées pour réaliser le diagnostic de gestation par échographie (E2, E4, E8) et les travaux sur surface fourragère (labours, semis et récolte $[\mathrm{E} 1, \mathrm{E} 2, \mathrm{E} 4, \mathrm{E} 5, \mathrm{E} 6])$. Toutes les exploitations (sauf celle gérée par le couple) emploient de 1 à 3 salariés permanents: le capataz (contremaitre) (E3, E6) qui a une certaine autonomie sur la conduite de la gestion de l'exploitation, et le peón (ouvrier agricole) (E1, E3, E4, E5, E6, E7, E8) qui exécute les tâches sous la direction de l'éleveur ou du capataz. La présence de salariés occasionnels est courante pour accomplir des tâches telles que l'entretien des clôtures fixes, le marquage et la castration des animaux (E3, E4, E5, E6, E7, E8).

\section{Travail d'astreinte (TA)}

Le travail d'astreinte correspond principalement aux recorridas, parcours de surveillance (Cittadini et al., 2001) qui s'effectuent généralement à cheval deux fois par jour. Pendant la recorrida se font les tâches de surveillance et d'intervention sur les animaux, de gestion du pâturage (changement de parc des animaux) et complémentation des animaux et même de contrôle et d'entretien des clôtures. La durée du parcours varie selon les périodes de l'année. Ainsi on distingue des périodes de mise bas au printemps, des périodes durant l'été où les traitements de lutte contre la " mouche de la corne "sont fréquents, et des périodes de surveillance classique (automnehiver). Les tâches étant imbriquées, la quantification du temps correspondant à la recorrida est donc globale sans répartition par atelier (ovin, bovin, ressources fourragères). Une seule exploitation (E5) irrigue pendant quelques semaines ses prairies semées. Le travail d'astreinte, calculé pour l'ensemble du collectif de travail, varie de 3 à 20 heures par jour avec une moyenne de 10 heures journalières (tableau 3).

\section{Travail de saison (TS) et travail rendu (TR)}

Quantifié en jours, le travail de saison concerne essentiellement les manipulations des animaux pour la gestion sanitaire - sevrage, marquage, castration, reproduction, pesage et préparation à la vente, ainsi que la tonte des ovins. Le temps passé à ces travaux dépend évidemment du nombre de bêtes, mais aussi des équipements pour les traitements sanitaires. Le travail sur la surface fourragère n'est important que dans les exploitations récoltant du foin (E4 et E5, cf. tableau 4). Dans les autres cas, ce travail est limité au semis des nouvelles prairies et varie selon les années. Le travail rendu chez d'autres éleveurs, en contrepartie pour l'entraide reçue, concerne trois exploitations (E2, E4, E7).

\section{Participation de la cellule de base}

L'étude de la participation de la cellule de base aux travaux d'astreinte ou de saison montre trois modalités d'organisation (tableau 5).

\section{Autonomie}

La cellule de base réalise seule les recorridas, et pour le travail de saison mobilise l'entraide plutôt que le salariat.

Cette modalité se rencontre dans les deux exploitations aux dimensions les plus faibles (E2, E4) : 150 hectares par personne de la cellule de base (pCB). La première est gérée par un couple pour lequel la recorrida constitue le coeur du métier et une façon de "travailler ensemble" (Fiorelli et al., 2007). La seconde est gérée par un double actif qui travaille en ville et se rend tous les jours sur son exploitation.

\section{Tableau 3. Travail d'astreinte (TA) annuel.}

Table 3. Annual routine work.

\begin{tabular}{lrrrrrrrr}
\hline Exploitation & E1 & E2 & E3 & E4 & E5 & E6 & E7 & E8 \\
\hline TA herbivore (heures/an) & 2637 & 2680 & 7237 & 1170 & 4739 & 4015 & 3373 & 2755 \\
TA herbivore/TA total (\%) & 100 & 100 & 100 & 100 & $94^{*}$ & 100 & 100 & 100 \\
TA moyen journalier (heures/jours) & 7,2 & 7,3 & 19,8 & 3,2 & 13,0 & 11,0 & 9,2 & 7,5 \\
TA herbivore/UGB (heures/UGB) & 10,0 & 8,3 & 5,1 & 10,4 & 9,1 & 10,5 & 7,8 & 5,8 \\
\hline
\end{tabular}

UGB : unité gros bétail ; $: 6 \%$ TA irrigation de surface fourragère.

Tableau 4. Travail de saison (TS) et travail rendu (TR).

Table 4. Seasonal work (TS) and counterpart work (TR).

\begin{tabular}{lrrrrrrrr}
\hline Exploitation & E1 & E2 & E3 & E4 & E5 & E6 & E7 & E8 \\
\hline TS total (journées/an) & 66 & 52,5 & 654 & 26,5 & 109,5 & 113 & 185 & 368,5 \\
Travail rendu (journées/an) & & 12 & & 2 & & & 12 & 100 \\
Proportion TS troupeau herbivore/TS total (\%) & 89 & 94 & 100 & 62 & 56 & 100 & 100 & 100 \\
Proportion TS surface fourragère/TS total (\%) & 11 & 6 & 0 & 38 & 44 & 0 & 0 & 0 \\
TS troupeau herbivore/UGB (journées par UGB) & 0,22 & 0,15 & 0,46 & 0,15 & 0,12 & 0,30 & 0,43 & 0,77 \\
\hline
\end{tabular}

UGB : unité gros bétail. 
Tableau 5. Modalités d'organisation du travail et temps disponible calculé (TDC).

Table 5. Work organizational modalities and available calculated time (TDC).

\begin{tabular}{|c|c|c|c|c|c|c|c|c|}
\hline \multirow[b]{2}{*}{ Exploitation } & \multicolumn{4}{|c|}{ Petits } & \multicolumn{3}{|c|}{ Moyens } & \multirow{2}{*}{$\frac{\text { Grand }}{\text { E3 }}$} \\
\hline & E4 & E2 & E1 & E7 & E5 & E6 & E8 & \\
\hline Syst. production bovins & Naiss. & Naiss. & Naiss. & Naiss. & $\mathrm{CC}$ & Naiss. & Naiss. & Naiss. \\
\hline Syst. production ovins & - & $\mathrm{CC}$ & $\mathrm{CC}$ & $\mathrm{CC}$ & $\mathrm{CC}$ & Naiss. & Naiss. & $\mathrm{CC}$ \\
\hline Hectares par pCB & 154 & 150 & 285 & 217 & 604 & 760 & 753 & 1861 \\
\hline Part du TA réalisée par la CB (\%) & 100 & 100 & 32 & 54 & 53 & 9 & 15 & 15 \\
\hline Part du TS réalisée par la CB (\%) & 46 & 52 & 30 & 54 & 29 & 15 & 14 & 15 \\
\hline Modalité d'organisation & \multicolumn{2}{|c|}{ Autonomie } & \multicolumn{3}{|c|}{ Partage } & \multicolumn{3}{|c|}{ Supervision } \\
\hline TDC/pCB (hr/pCB/an) & 1445 & 1258 & 1680 & 1411 & 335 & 2076 & 1802 & 1188 \\
\hline
\end{tabular}

Naiss. : naisseur uniquement ; CC : cycle complet ; $C B$ : cellule de base ; $\mathrm{pCB}$ : personne de la cellule de base.

\section{Partage}

La cellule de base réalise avec un salarié permanent les recorridas et le travail de saison non délégué aux entreprises.

Cette modalité concerne E1 et E7 (250 hectares par pCB environ) et E5 (600 hectares par pCB). La première exploitation est gérée par un éleveur âgé ayant d'autres sources de revenu (location de maison) : il se réserve la recorrida du matin. L'exploitation E7 est gérée par le père et le fils. Le père, âgé, prépare son départ et ne participe pas aux recorridas. Pour la troisième exploitation (E5), la volonté de maîtrise technique de son système d'élevage, conduit l'éleveur à rester dans une configuration de partage malgré l'importance des dimensions.

\section{Supervision}

La cellule de base ne participe pas ou que très peu aux travaux sur l'exploitation qui sont délégués aux salariés et aux entreprises pour les chantiers saisonniers. Elle réalise le contrôle et la supervision des activités par entretien téléphonique, visites régulières ou participation modérée.

Cette modalité concerne les exploitations les plus grandes (E3, E6, E8). La maind'œuvre salariée est de règle. Ce mode d'organisation permet au premier exploitant (E6) de se consacrer à la vie politique. Pour le deuxième exploitant, âgé, c'est une configuration qui lui permet de transmettre progressivement l'exploitation à ses enfants qui travaillent comme salariés. Dans le cas de l'exploitation la plus grande (E3), l'organisation s'appuie sur le capatazà qui l'exploitant fait confiance pour l'exécution du travail, tout en se réservant une plus grande participation dans le travail de saison de commercialisation, de contrôle des pesées et de tri des animaux.

\section{Temps disponible calculé}

Le temps disponible calculé (TDC) est un indicateur de la marge de manouvre en temps dont dispose la cellule de base. Il doit être considéré par rapport à la situation de cette cellule de base et à ses choix de gestion de l'exploitation.

Pour notre échantillon, les valeurs de cet indicateur témoignent, pour 6 cas sur 8, de situations relativement confortables (de 1100 à 1800 heures par personne et par an) par rapport à nos références françaises (Pichereau et al., 2004 ; Chauvat et Cournut, 2009). Deux cas extrêmes sont à noter : l'exploitation E6 avec un TDC plus que confortable (2 076 heures) et l'exploitation E5 présentant, au contraire, une situation qu'on qualifierait de tendue avec un TDC de 335 heures.

Ces deux exploitations ont des surfaces comparables (600 et 700 hectares) et sont toutes les deux gérées par un exploitant, mais avec des différences importantes en termes de choix de système: simplifié (uniquement naisseur) et très extensif (chargement faible de 0,5 UGB par hectare) pour l'exploitation E6, plus sophistiqué et intensif pour l'exploitation E5 qui engraisse tous ses animaux, a une surface en prairies semées et irriguées, confectionne un stock de foin et a un cheptel bovin deux fois plus important que celui de l'exploitation E6.

Les choix d'organisation du travail sont eux aussi bien différents, en lien avec les choix de vie de ces deux exploitants. Le premier (E6) a choisi de vivre en ville, de déléguer le travail de l'exploitation à ses salariés et à des entreprises pour les chantiers saisonniers, et de ne conserver qu'un rôle minimum de supervision et décision dans la gestion de l'exploitation. Cette stratégie entrepreneuriale fréquente en Amérique du Sud lui permet de se consacrer à ses activités politiques. Le second éleveur vit sur son exploitation et s'investit dans son fonctionnement. Il témoigne d'une volonté de maitrise technique de son élevage et d'intensification de sa conduite. L'exploitation est mécanisée (tracteur et charrue), ce qui est exceptionnel dans notre échantillon. Il garde un investissement personnel fort dans la réalisation des tâches.

\section{Discussion}

\section{Principaux éléments de caractérisation de l'organisation du travail}

L'analyse du travail dans cet échantillon permet une meilleure connaissance du fonctionnement des exploitations d'élevage extensif uruguayen. Fondé sur la recorrida, le travail d'astreinte présente des caractéristiques proches de ce que l'on rencontre en France dans des élevages bovin viande (Pichereau et al., 2004) : biquotidien, d'une durée de 3 à 6 heures par jour et par personne, avec efficience de 5 à 10 heures par UGB. La nature du travail diffère en revanche largement. En Uruguay, c'est le travail traditionnel du gaucho, en plein air, ne nécessitant 
d'autre équipement qu'une monture, et englobant des tâches variées concernant tant les bovins que les ovins (soins, déplacements, alimentation, mise bas...), que les prairies et les clôtures (contrôle, entretien...). Les périodes d'astreinte sont marquées par les mises bas et les traitements sanitaires pour la mouche de la corne. Le travail de saison, caractéristique d'un élevage herbivore extensif fondé sur des pairies naturelles, nécessite peu d'installation (parcs de contention) mais une main-d'œuvre importante pour réaliser des chantiers concernant un nombre élevé d'animaux.

Les modalités d'organisation du travail dans ces élevages montrent un recours au salariat systématique pour le travail de saison et très fréquent dans le travail d'astreinte dès que la charge pesant sur les éleveurs dépasse 150 hectares par personne. En France, le seuil d'emploi d'un salarié permanent en élevage allaitant est également de cet ordre (Pichereau et al., 2004). La délégation des travaux de saison aux entreprises est aussi typique de ces organisations, de par l'absence de matériel sur les exploitations et la nécessité d'être à plusieurs pour manipuler les animaux. Le bénévolat et l'entraide n'ont pas autant de poids qu'en France (Chauvat et Cournut, 2009) ; quand ils existent, c'est plutôt pour le travail de saison (marquage, traitements sanitaires ou castration).

\section{Lien entre dimension des exploitations, système de production, et modalités d'organisation du travail}

Dans les petites exploitations, le recours au salariat (péon) est souvent limité et l'éleveur participe à la réalisation des travaux d'astreinte ou de saison, ce qui, en plus de réduire les coûts, lui garantit aussi la mâtrise technique de la conduite. Dans ces situations, l'introduction de pratiques plus complexes (réalisation de prairies semées, confection de foin...) est facilitée.

Dans les exploitations les plus grandes, la délégation des travaux est forte et le contrôle de l'éleveur sur la conduite technique plus faible : il s'appuie sur la maind'œuvre salariée permanente. Ce lien entre dimension, système de production et modalité d'organisation du travail n'est pas absolu ; ainsi, dans de grandes exploi- tations, l'éleveur peut concilier grande dimension, recours important à du salariat, et maîtrise technique d'une conduite complexe, en s'investissant fortement dans la gestion de son équipe de travail (Levrouw et al., 2007 ; Cittadini et al., 2001). D'autres facteurs liés à l'éleveur et à ses attentes en termes de travail doivent également être pris en compte : travailler avec sa femme, transmettre son expérience à ses enfants (Perrachón, 2009), se réserver du temps pour d'autres activités (Malaquín, 2009), améliorer la productivité de son exploitation...

\section{Application de la méthode Bilan travail}

L'application de la méthode nous a conduits à modifier le contour de la cellule de base pour prendre en compte la combinaison d'activités des exploitants comme le font Madelrieux et Dedieu (2008), et à considérer le travail de supervision et d'organisation comme faisant partie du travail d'astreinte. Ces adaptations nécessaires pour rendre compte de certaines situations de l'élevage uruguayen sont aussi à considérer dans des situations émergentes en France où la main-d'œuvre (salariés ou associés) est devenue importante. Elles montrent les limites de la méthode Bilan travail lorsque la personne enquêtée n'est pas elle-même très engagée dans la réalisation pratique des travaux: l'évaluation des temps de travaux réalisés par les salariés perd alors en fiabilité. Elles modifient aussi la signification de la marge de manœuvre en temps qui devra être recalibrée en Uruguay avec cette nouvelle définition. Les prochaines applications de la méthode en Uruguay devront permettre de poursuivre la construction de références locales et mettre en correspondance les indicateurs de résultats avec le ressenti des éleveurs sur leur charge de travail.

\section{Conclusion}

Cette étude à caractère exploratoire confirme tout l'intérêt de la prise en compte du travail dans l'analyse du fonctionnement des exploitations d'élevage. L'application de la méthode Bilan travail sur notre échantillon d'exploitations a montré une diversité de situations que nous avons pu décrire et expliquer.
Ce nouvel éclairage des élevages nous permet de mieux comprendre les logiques de fonctionnement et les déterminants qui les sous-tendent, et ainsi d'adapter le conseil technique. Molina et Alvarez (2009) avaient déjà montré que le travail était un des éléments pris en compte parmi les raisons de nonadoption par les éleveurs des nouvelles pratiques techniques promues par les services de développement pour améliorer la productivité des élevages. Cette étude donne des pistes pour la prise en compte du travail dans le conseil technique aux éleveurs. Nous devrons non seulement nous donner les moyens d'évaluer les conséquences de changements techniques sur l'organisation du travail, mais comprendre aussi sur quels piliers fondateurs repose l'organisation de chaque exploitation (travailler avec sa femme, double activité...).

\section{Remerciements}

Cette étude a été réalisée dans le cadre du projet TRANS (transformations de l'élevage et dynamique des espaces) financé par l'Agence nationale de la recherche (ANR, Agriculture et développement durable). Les auteurs remercient Gérard Servière et Sophie Chauvat (Institut de l'élevage) pour leur aide.

\section{Références}

Arbeletche P, Carballo C. Dinámica agrícola y cambios en el paisaje. Consejo Europeo de investigaciones sociales de América Latina (CEISAL) V Simpossium, Belgique, 2008. www. reseau-amerique-latine.fr/ceisal-bruxelles/ESE/ ESE-6-arbeletche-carballo.pdf

Cittadini R, Dedieu B, Derail L, Perez R. Trabajo y tecnología en explotaciones ganaderas de la provincia de Buenos Aires. In: Neiman G, ed. Trabajo de campo. Producción, tecnología y empleo en el medio rural. Buenos Aires : ediciones CICCUS, 2001. www.inta.gov.ar/balcarce/ info/documentos/econo/rural/cittadini.PDF

Chauvat S, Cournut S. Synthèse de 600 Bilans Travail dans 7 filières herbivores et monogastriques. Rencontres nationales sur le travail en élevage, 2009. www.inst-elevage.asso.fr/html1/IMG/ pdf No 2 Referentiels travail.pdf

Cournut S, Jordan A, Dedieu B, Servière G. Guide méthodologique pour l'analyse de groupe de "Bilans Travail " en exploitations d'élevage. Collection Institut de l'élevage, Casdar - Trans. 2009. www1.clermont.inra.fr/add-trans/pdf/guidebilantravailcournut.pdf 
Dedieu B, Servière G, Madelrieux S, Dobremez L, Cournut $S$. Comment appréhender conjointement les changements techniques et les changements du travail en élevage? Cah Agric 2006 ; 15: 506-13. Doi : $10.1684 /$ agr.2006.0028

Estadísticas Agropecuarias (DIEA), Ministerio de Ganadería Agricultura y Pesca. Contribucion al conocimiento de la ganadería mediante la declaración jurada de DI.CO.SE. In : Pereira G, ed. Trabajos especiales $n^{\circ} 17$. Montevideo (Uruguay): Ministerio de Ganadería Agricultura y Pesca (Uruguay), 2000. www.mgap.gub.uy/portal/ agxppdwn.aspx?7,1,89,0,S,0,1049\%3bS\%3b2\%3 b120.

Fiorelli C, Dedieu B, Pailleux JY. Explaining diversity of livestock-farming management strategies of multiple-job holders: importance of level of production objectives and role of farming in the household. Animal 2007; 1 : 1209-18.
Levrouw F, Morales $\mathrm{H}$, Arbaletche $\mathrm{P}$, Malaquin I, Tourrand JF, Dedieu B. Les élevages uruguayens, le long terme et les incertitudes: une diversité de stratégies pour se maintenir dans la production. Renc Rech Rum 2007; 14 : 413-6.

Malaquin I. Ganadería a tiempo parcial y pluriactividad de la unidad familiar. In: Dieguez F, Morales $\mathrm{H}$, eds. Familias y campo. Rescatando estrategias de adaptación. Montevideo (Uruguay) : Instituto Plan Agropecuario, 2009.

Maldelrieux S, Dedieu B. Qualification and assessment of work organisation in livestock farms. Animal 2008 ; 2: 435-47.

Molina C, Alvarez J. Identificacion de factores incidentes en las decisiones de adopcion de tecnologia en ganaderos criadores familiares. Agrociencia 2009; 13: 70-83. www.fagro.edu. uy/agrociencia/VOL13/2/agrociencia\%20diciembre \%202009.pdf.
Perrachón J. Sucesión generacional en empresas familiares agropecuarias. In: Dieguez $F$ Morales $\mathrm{H}$, eds. Familias y campo. Rescatando estrategias de adaptación. Montevideo (Uruguay) : Instituto Plan Agropecuario, 2009.

Pichereau F, Becherel JP, Farrie JP, et al. Fonctionnement des grands troupeaux de vaches allaitantes: analyse des déterminants structurels et techniques de I'organisation du travail. Renc Rech Rum 2004 ; 11 : 129-36.

Riella A, Mascheroni P. Una nueva mirada sobre los territorios rurales: trabajo no agrícola y pluriactividad en el Uruguay rural. In: Chiappe M, Fernández E, Carámbula E, eds. El campo uruguayo. Una mirada desde la sociología rural. VII Coloquio de Transformaçoes Territoriais. Montevideo (Uruguay) : Facultad de Agronomía, 2008. 